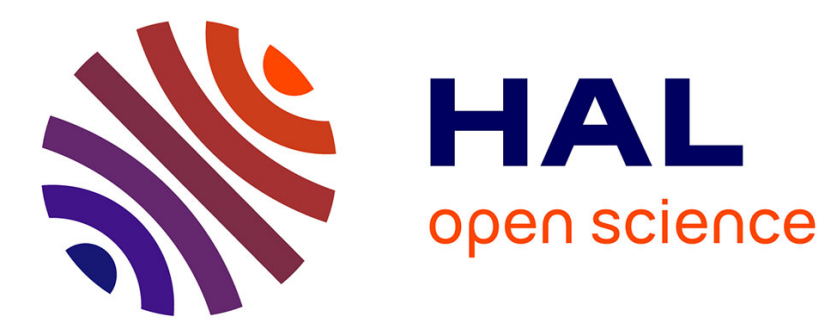

\title{
Power analysis for the design of a large area ultrasonic tactile touch panel
}

Yi Yang, Betty Lemaire-Semail, Frédéric Giraud, Michel Amberg, Yuru

Zhang, Christophe Giraud-Audine

\section{- To cite this version:}

Yi Yang, Betty Lemaire-Semail, Frédéric Giraud, Michel Amberg, Yuru Zhang, et al.. Power analysis for the design of a large area ultrasonic tactile touch panel. European Physical Journal: Applied Physics, 2015, 72 (1), pp.11. 10.1051/epjap/2015150051 . hal-01198587

\section{HAL Id: hal-01198587 \\ https://inria.hal.science/hal-01198587}

Submitted on 14 Sep 2015

HAL is a multi-disciplinary open access archive for the deposit and dissemination of scientific research documents, whether they are published or not. The documents may come from teaching and research institutions in France or abroad, or from public or private research centers.
L'archive ouverte pluridisciplinaire HAL, est destinée au dépôt et à la diffusion de documents scientifiques de niveau recherche, publiés ou non, émanant des établissements d'enseignement et de recherche français ou étrangers, des laboratoires publics ou privés. 


\title{
Power Analysis for the Design of a Large Area Ultrasonic Tactile Touch Panel
}

\author{
Yi Yang ${ }^{1 \mathrm{a}}$, Betty Lemaire-Semail ${ }^{2}$, Frédéric Giraud ${ }^{2}$, Michel Amberg $^{2}$, Yuru Zhang ${ }^{1}$, and Christophe Giraud-Audine ${ }^{2}$ \\ 1 State Key Laboratory of Virtual Reality Technology and Systems, Beihang University, 37 Xueyuan Road, 100191 Beijing, \\ China. \\ 2 L2EP-IRCICA, University Lille 1, 50, Avenue Halley, Parc Scientifique de la Haute Borne, 59650 Villeneuve d'Ascq, France
}

Received: date / Revised version: date

\begin{abstract}
Tactile interfaces are intuitive but lack of haptic feedback. One method to provide tactile feedback is to change the friction coefficient of the touch surface. Several small-size tactile devices have been developed to provide programmable friction coefficient based on the squeeze air film effect. This effect is produced by ultrasonic vibration of the tactile plate thanks to piezoceramics. In order to design larger embedded tactile feedback areas, a key issue is the power consumption. In this paper, we present the power analysis of a tactile device which is based on the squeeze film effect. We first investigate the source of power consumption by a series of measurements. Then, an analytical model is developed to estimate the power, which gives the conclusion that, when the vibration amplitude is constant, the power consumption is not related to the number of piezoelectric actuators. According to this result, we design a large area $(198 \mathrm{~mm} \times 138 \mathrm{~mm})$ tactile plate with only eight piezoelectric actuators. Experimental results show that the power consumption of the large tactile plate is less than 2 Watts. Moreover, we also find that the power consumption of the large tactile plate was predictable with the measurement results from small plates with an average error of less than $10 \%$.
\end{abstract}

\section{Introduction}

Touch screen interaction is prevalent nowadays. Due to their directness and intuitiveness, touch screens have been widely applied to consumer electronics, public kiosks, and automatic teller machines. However, a significant disadvantage of touch screens is their lack of haptic feedback $[1,2]$. Users have to rely on their vision to interact with the touch screen. This may increase users' visual workload and the risk of making errors [3].

Several types of haptic interfaces have been developed to improve touch interaction. A majority of them use vibration motors [4], voice coils [3] or solenoid actuators [5] to vibrate the whole tactile interface. Others use piezoelectric actuators $[6,7]$ or ultrasonic vibrators [8] to vibrate the touch screen in order to realize a button-click feedback. Some technologies are also applied to change the surface properties. Jansen, Karrer and Borchers [9] developed the MudPad, which changes the hardness of the touch surface using electromagnet-actuated magnetorheological (MR) fluid. Harrison and Hudson [10] developed a

\footnotetext{
${ }^{a}$ This work has been carried out within the framework of the INRIA Mint Project, and the Stimtac project of the IRCICA (Institut de Recherche sur les Composants logiciels et matériels pour l'Information et la Communication Avancée). This work has also been supported by the State Key Laboratory of Virtual Reality Technology and Systems in Beihang University.
}

device that can "pop out" pneumatic actuated deformable buttons. Nevertheless, all these solutions suffer either from a lack of sensitivity to reproduce thin textures or from the difficulties to extend the technology to large tactile feedback surfaces.

An alternative method to provide tactile feedback on a large surface is to change the roughness of the touch pannel. For example, the TeslaTouch [11] increases surface apparent roughness by means of producing electrostatic force. While this technology was highly scalable, it still needs high voltage supply to reach performant tactile rendering, and the user needs to be connected to the ground. On the other hand, Kotani, Takasaki and Mizuno created a transparent tactile device with a slider-based interface using ultrasonic $(15 \mathrm{MHz})$ surface acoustic wave [12]. The periodic contact between the slider and the touch surface decreases the perceived surface roughness when the user moves the slider. However, this device can not be operated directly with a bare finger because of the thermal effect which occurrs in that case.

Surface roughness can also be changed by using the squeeze air film effect: the air in the small gap between a finger and a plate moving perpendicular to the finger at a high frequency will be compressed before it escapes from the gap. If the vibration frequency is high enough and the mean clearance between the plate and the fingers is small enough, the trapped air in between will result in an average pressure higher than the atmospheric pressure. 
Thus, an air cushion which is able to reduce the apparent friction coefficient between the finger and the plate is produced. It has been shown that the friction reduction is in proportion to the vibration amplitude between given limits [13]. Several devices have been developed based on the squeeze air film effect. They are all working at ultrasonic frequencies, and often at the resonant frequency of the whole device. Some work with piezoelectric actuators that are glued all over the underside of the devices [13-15]. Some work with actuators be transparent for applications as touch screens $[16,17]$. By modulating the vibration amplitude as a function of the finger position, these devices are attached only at two edges so that the middle part of the device can be transparent for applications as touch screens $[16,17]$. By modulating the vibration amplitude as a function of the finger position, these devices are notably used to simulate fine textures $[15,18]$ and facilitate pointing tasks $[19,20]$. However, a limitation of these devices is that their workspace is small (as shown in Table 1), as compared with the requirement of supporting multi-touch interaction and large display touch interfaces (e.g., the IPad). Until recently, Mullenbach et al. incorporated a variable friction surface (TPad) and a Kindle FireTM tablet to realize tactile feedback on a relatively larger touch screen [21].

The development of a large area tactile interface based on the squeeze film effect is challenging. One main issue is the power consumption. Indeed, the dissipated power converts to heat which increases the temperature of the touch surface making the user uncomfortable. Moreover, the heat also changes the resonance frequency of the plate, reducing the vibration amplitude and further degrading the perception of tactile feedback. Furthermore, great power consumption needs powerful power supply which is not applicable to portable mobile devices. In our previous research, we found that the power consumption could be reduced by using thin resonator and thin piezoelectric actuators [22]. These studies on power consumption were carried out on a device with piezoelectric actuators all over the underside of the touch surface. This device is the STIMTAC display from Université Lille1 [23]. Recent research proposes to glue piezoelectric actuators only at the edges of the device in order to provide maximum transparent workspace at the center of the touch surface and to reduce the number of piezo-actuators [17]. However, there is little research on how the number and layout of the piezo-actuators affect the power consumption of such devices [24]. In this paper, we develop an approach in order to plan the power consumption of the piezoelectric vibrating tactile feedback devices. First, a model taking into account the number of piezoelectric actuators is established to calculate the power consumption. Based on this model, an experimental study regarding their layout is achieved. By this way, we may offer guidelines on how to use minimum piezoelectric actuators to develop a large area tactile feedback device operating with squeeze film effect.

In the following sections of the paper, we first present a series of measurements that are carried out to investigate
Table 1. The touch area of the present variable friction tactile devices

\begin{tabular}{ll}
\hline Device & Touch Area \\
\hline T-Pad [15] & $\emptyset 25 \mathrm{~mm}$ \\
LATPad [16] & $76.2 \mathrm{~mm} \times 76.2 \mathrm{~mm}$ \\
TPad Fire [21] & $165 \mathrm{~mm} \times 130 \mathrm{~mm}$ \\
STIMTAC [18] & $83 \mathrm{~mm} \times 49 \mathrm{~mm}$ \\
Transparent STIMTAC [17] & $93 \mathrm{~mm} \times 65 \mathrm{~mm}$ \\
\hline
\end{tabular}

the power consumption of the STIMTAC device (Section 2 ). Then we developed an analytical model to estimate the power consumption (Section 3). The main result of this analysis is that the power consumption is not related to the number of piezoelectric actuators but related to their layouts when the vibration amplitude was constant. According to this result, we designed a large area tactile plate with very few piezoelectric actuators (Section 4). Measurement results show that the power consumption of the large tactile plate is less than 2 Watts. And the power consumption is well estimated by the analytical model with an average error less than $10 \%$.

\section{Power consumption measurements}

In previous researches in Univeristy Lille1, the geometric parameters of the tactile plate have been optimized[25]. We noted that the power consumption of the plate was in proportion to the thickness of both the resonator and the piezoceramics $[22,25]$. However, these analyses were performed on the device whose resonator was fully glued with piezoceramics. So far, neither the number nor the layout of the piezoceramics was a variable parameter. In this study, we want to investigate the influence of these parameters on the power consumption. Therefore, we first conducted a series of measurements to reveal the major source of the power consumption. The tactile plate consists of three main parts: the resonator, the piezoceramics, and the glue bonding. Considering these components, we assume that the power consumption of the tactile plate includes three parts: mechanical power losses, electrical power losses and electro- mechanical power losses. The mechanical power losses may be due to the vibration of the whole device (including the resonator, the piezoceramics and the glue). The electrical power losses may come from the hysteresis losses in dielectric; and the electro-mechanical power losses come from the hysteresis cycle in the piezoelectric energy coupling [26]. In the following measurements, we investigate the source of the power consumption by analyzing each possibility.

It should be noted that the objective of conducting these measurements is to find the main source of power consumption. It was difficult and out of the scope of this paper to measure the exact value of each power component. Therefore, we only take into account the main power resource and develop a model to analyze the power consumption of the device. 


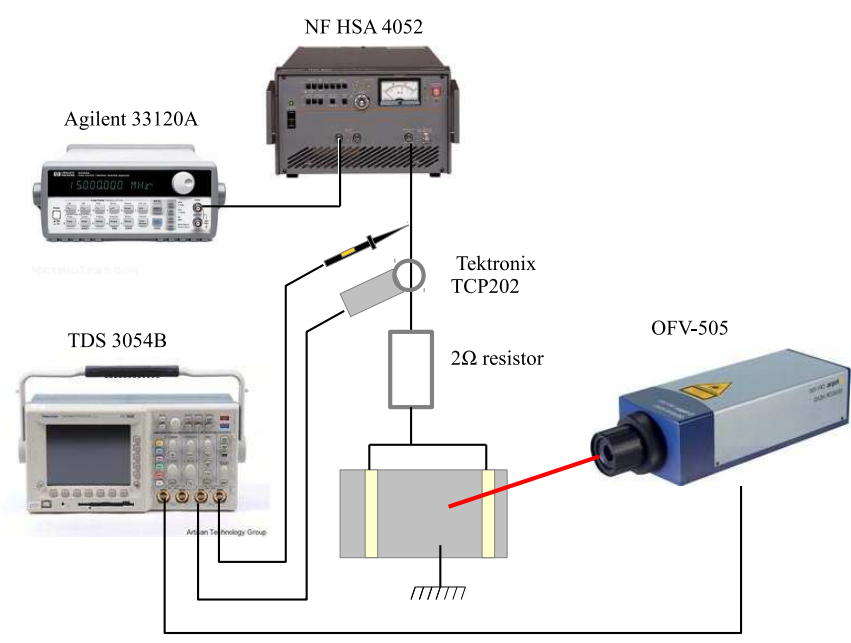

Fig. 1. Schematic of the power measurement

\subsection{Measurement setup}

In the measurements, we recorded the applied voltage, the vibration velocity and the power consumption. The figure 1 shows a schematic of the power measurement.

We used a waveform generator (Agilent 33120A) to generate sinusoidal wave voltage. The frequency of the sinusoidal signal was set at the resonance frequency of each tactile plate we analysed. The sinusoidal signal was amplified 20 times by a high speed amplifier (NF HAS 4052) to actuate the tactile plate. The vibration velocity was measured by a single-point LaserDoppler Vibrometer (Model OFV-505, Polytec GmbH, Waldron, Germany). The current flowing through the piezoceramics was sensed by a current probe (Tektronix TCP202). The voltage, current and vibration speed images were recorded through an oscilloscope (Tektronix TDS 3054B). The oscilloscope was also in charge of computing the electric power consumption. To do so, after careful DC cancellation and noise reduction through averaging, the product between instant voltage and instant current was computed and displayed using a MATH channel. The average value - over an integer number of periods - of that product was considered to be the supplied power. A $2 \mathrm{ohm}$ shunt resistor was put in serial with each tactile device in order to measure the supplied power. The current was very sinusoidal and the power taken by the resistor was at most $10 \%$ of the total supplied power. Therefore, we assume that the effect of the resistor was negligible.

In the measurements, each piezoceramic column contained 4 piezoceramics (NCE 41, Noliac Inc.). The dimension of the piezoceramics was: $5 \mathrm{~mm} \times 9 \mathrm{~mm} \times 0.5 \mathrm{~mm}$. Material details of the piezoceramics can be found in [27]. A list of the materials is presented in Table 2. All plates worked at the $(9,0)$ mode shape of a standing wave. During the measurements, the tactile devices were placed on a flat cushion in order to realize an all-free boundary condition.
Table 2. Material parameters of the tactile plate

\begin{tabular}{lll} 
Mechanical properties of the aluminum resonator \\
\hline Young's module & $E_{i}$ & $67109 \mathrm{~N} / \mathrm{m}^{2}$ \\
Poisson's ratio & $\nu_{i}$ & 0.34 \\
Density & $\rho_{i}$ & $2790 \mathrm{~kg} / \mathrm{m}^{3}$ \\
\multicolumn{4}{l}{ Mechanical properties of $\mathrm{NCE} 41$ piezoceramics $[27]$} \\
Charge constant & $d_{31}$ & $-13010^{-12} \mathrm{C} / \mathrm{N}$ \\
Elastic constant & $c_{11}^{E}$ & $8010^{9} \mathrm{~N} / \mathrm{m}^{2}$ \\
Poisson's ratio & $\nu_{p}$ & 0.33 \\
Density & $\rho_{p}$ & $7900 \mathrm{~kg} / \mathrm{m}^{3}$ \\
\hline
\end{tabular}

\subsection{Electric power losses}

Method: We assumed that the mechanical power losses were proportional to the square of the vibration velocity, and the electric power losses were proportional to the square of the applied voltage, according to [26]. Piezoelectric actuators were always treated like capacitors and the electric power losses of capacitors were calculated as dielectric power losses [28]. To study the changes of dielectric power losses, we kept the mechanical power losses constant through keeping the same vibration velocity. Firstly, we record the vibration velocity at resonance as $v_{0}$ and the corresponding power as $P_{0}$. Then we slightly changed the frequency of the applied voltage apart from the resonance frequency without changing the mode shape. As a consequence, the vibration velocity decreased. Then, we applied a higher voltage to regain the vibration velocity $v_{0}$ and record the power needed at this state as $P_{t}$. If $P_{t}$ does not increase significantly, we can deduce that the dielectric power losses are negligible.

Results: The experiment was carried out on the three plates of Fig. 2 with 1 column, 4 columns and 9 columns of piezoceramics. These three plates have different number and layout of piezoceramics. Nevertheless, the width of a piezoceramic is always close to the half wavelength of the deformation mode shape $\lambda / 2$. We found that decrease or increase frequency symmetrically to the resonance implied similar results. Therefore we only present the power changes through increasing the frequency. Accordingly, we record $P_{t}$ when the vibration velocity at resonance was regained after it was decreased to $80 \%, 60 \%$ and $50 \%$ of the value at the resonance frequency. We applied three vibration velocity levels $(0.125 \mathrm{~m} / \mathrm{s}, 0.25 \mathrm{~m} / \mathrm{s}$, and $0.375 \mathrm{~m} / \mathrm{s})$ in the measurements for the two first plates and two for the third one $(0.125 \mathrm{~m} / \mathrm{s}, 0.25 \mathrm{~m} / \mathrm{s})$ because we could not supply power with enough voltage.

Experimental results are presented in Fig. 2. According to the first two measurements (Fig.2 (a) and (b)), we find that the power $P_{t}$ does not increase significantly. This indicates that the dielectric power losses are negligible. To further confirm this conclusion, we increased the frequency supply of the 1 column plate (from $37180 \mathrm{~Hz}$ to $37560 \mathrm{~Hz}$ ) so that much higher voltage was applied to regain the vibration velocity in that case. We intended to check if $P_{t}$ increased significantly in this process. As shown in Fig.2(c), we found that $P_{t}$ only increased from $100 \mathrm{~mW}$ 


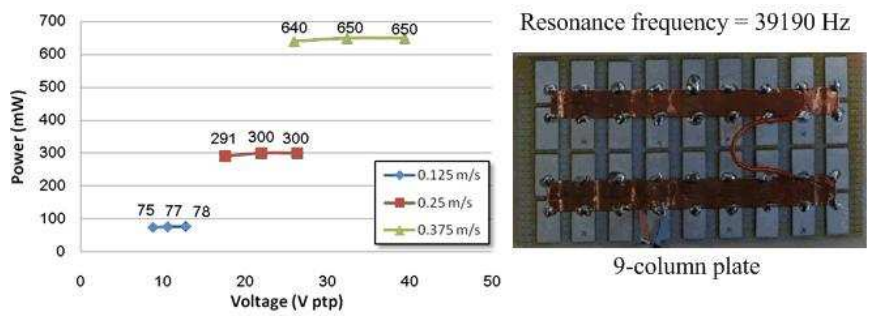

(a) Power changes in the 9 columns plate

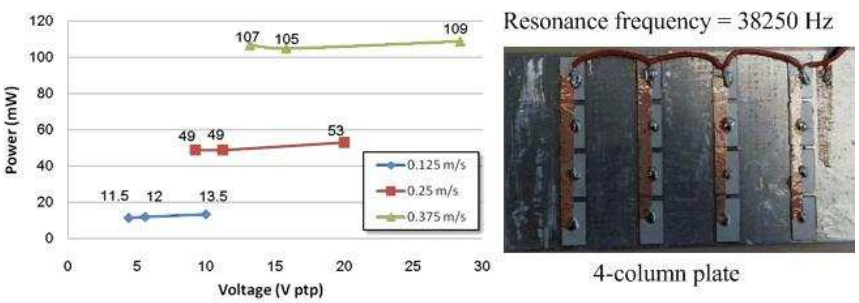

(b) Power changes in the 4 columns plate

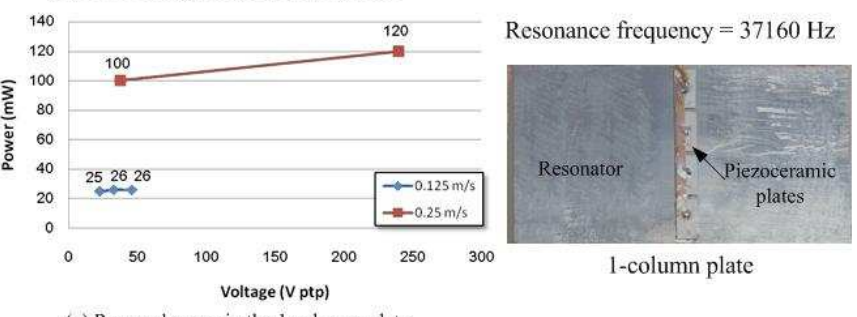

(c) Power changes in the 1 columns plate

Fig. 2. Power changes in the three tactile plates. Piezoceramics were glued on the resonator to excite the resonance vibration. The dimensions for the aluminum resonator were $76 \mathrm{~mm} \times 41 \mathrm{~mm} \times 1 \mathrm{~mm}$.

to $120 \mathrm{~mW}$ when voltage was increased from $37.6 \mathrm{~V}$ to $240 \mathrm{~V}$. We therefore infer that the dielectric power losses are only noticeable when applied voltage is high. However, in our application, we do not apply voltage above $100 \mathrm{~V}$. Therefore, we consider that the dielectric power losses are negligible in our tactile plates.

\subsection{Mechanical power losses}

\subsubsection{Mechanical power losses from the resonator}

Method: In the second step, we analyze mechanical power losses due to the vibration of the resonator. We intend to check if they are due to the mechanical damping of the resonator. In this experiment, we compare the power consumption when changing the size of the resonator. In order to minimize the influence of the glue and the piezoceramics, we used the 1 column plate (as shown in Fig.2). Then we cut certain lengths from each edge of the resonator symmetrically in each measurement. We conducted three measurements with the lengths of the resonator shortened from $75 \mathrm{~mm}$ to $59 \mathrm{~mm}$, and finally $28 \mathrm{~mm}$. We applied the same voltage, $20 \mathrm{~V}$ peak-to-peak (ptp), to the piezoceramics and measured the power and vibration velocity at the center of the plate.

Results: Measurement results are listed in Table 3. We found that the size of the resonator did not change
Table 3. Measurement results according to the length of the resonator

\begin{tabular}{lccc}
\hline Size & $75 \times 41$ & $59 \times 41$ & $28 \times 41$ \\
nb of antinodes & 9 & 7 & 3 \\
\hline Frequency $(\mathrm{Hz})$ & 37160 & 37180 & 35130 \\
Voltage $(\mathrm{V}, \mathrm{ptp})$ & 20 & 20 & 20 \\
Power $(\mathrm{mW})$ & 27 & 23 & 25 \\
Velocity $(\mathrm{m} / \mathrm{s}, \mathrm{ptp})$ & 0.175 & 0.188 & 0.188 \\
\hline
\end{tabular}

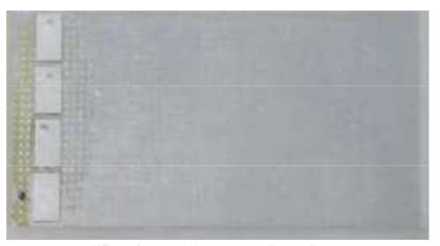

(a) One column at the edge

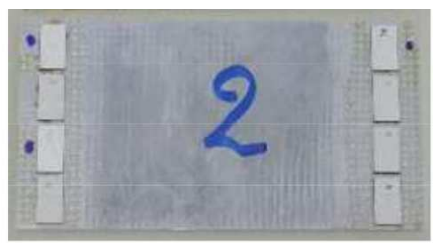

(b) Two columns at the edges
Fig. 3. Two plates with piezoceramics glued at the edges. The dimensions for the aluminum resonator were $76 \times 41 \times 1.2 \mathrm{~mm}$.

the power nor the velocity significantly. Therefore we infer that the resonator does not contribute to the mechanical power losses. In fact, the vibration velocity was higher at the edges than at the centre of the 1-column plate(which is the position of an antinode of vibration). We cut off the edges of the resonator and measured at the center. If the edges of the resonator cost power, then the changes of power should be significant. However, we did not notice this effect in our measurement. This inference confirmes us that the power losses are not due to the damping of the resonator.

\subsubsection{Mechanical power losses from the piezoceramics}

Method: According to our aforementioned measurements, we found that the power losses were neither due to the dielectric power losses nor to the vibration of the resonator. Then, we supposed that the power losses were caused by the piezoceramics. The mechanical losses of the piezoceramics may be due to the elastic losses [29] or the damping in the glue bonding between the piezoceramics and the resonator. To analyze the mechanical power losses, we made a tactile plate with one column of piezoceramics and compared it with a 2-column plate, as shown in Fig.3. The dimension of the aluminum resonator were $76 \times 41 \times 1.2 \mathrm{~mm}$ while that of the piezoceramics was $5 \times 9 \times 0.5 \mathrm{~mm}$.

Results: Measurement results are presented in Table 4. Let us first analyse the two left columns: in both cases, the same voltage is applied to the same number of piezoceramics, but for the second plate, there are also other piezoceramics which are vibrating although not supplied. If there were mechanical power losses in the piezoceramics, as the vibration amplitude is the same, the power consumption of the 2-column plate should be twice of the 1column. However, we found that both the power and the vibration velocity were similar in these two conditions. This result indicates that the unpowered column of piezoceramics did not take power. So the mechanical power 
Table 4. Measurement of the 1-columns and 2 columns plates.

\begin{tabular}{lccc}
\hline plate & 1-column & $\begin{array}{c}\text { 2-columns } \\
\text { 1 powered }\end{array}$ & $\begin{array}{c}\text { 2-columns } \\
\text { 2 powered }\end{array}$ \\
\hline Frequency $(\mathrm{Hz})$ & 43792 & 44810 & 44720 \\
Voltage $(\mathrm{V}, \mathrm{ptp})$ & 24 & 24 & 12 \\
Power $(\mathrm{mW})$ & 115 & 112 & 100 \\
Velocity $(\mathrm{m} / \mathrm{s}, \mathrm{ptp})$ & 0.3 & 0.26 & 0.27 \\
Amplitude $(\mu \mathrm{m}, \mathrm{ptp})$ & 1.08 & 0.92 & 0.96 \\
\hline
\end{tabular}

losses of piezoceramics were negligible. Now let us consider the results of the right column in table 4: the number of powered piezoceramics has been multiplied by two in comparison with the two first ones; as a result, half the voltage is necessary to obtain the same vibration amplitude. Other experiments have been performed to confirm this result. Namely, the vibration amplitude was in proportion to the number of powered piezoceramics and the applied voltage:

$$
A \propto n_{c} V
$$

where $A$ is the vibration amplitude, $n_{c}$ is the number of powered piezoceramics, and $V$ is the applied voltage. Moreover, once again, the power consumption for this last plate was similar at a given vibration amplitude, in agreement with the assumption that the mechanical losses inside piezoceramics are neglectable.

\subsection{Summary}

According to our measurements, we found that the power losses of the tactile plate were mainly due to the powered piezoceramics. Dielectric power losses and the mechanical power losses can be negligible when analyzing the power losses. Since these factors were excluded, we supposed that the power losses were mainly due to the hysteresis losses within piezoelectric coupling. Based on this assumption, we developed an analytical model to analyze the power losses due to hysteresis effect in piezoelectric energy conversion process.

\section{Power consumption modeling}

\subsection{General asumption}

According to our measurement results, we concluded that the power consumption was mainly due to the electromechanical conversion. Therefore, we assume that the electrical power provided to the device is fully dissipated into the piezoelectric layer. Moreover, the power losses due to acoustic radiation are assumed to be negligible in comparison with the other losses.

In the following parts, we will analyze the deformation of the piezoceramics and calculate the power consumption of the tactile device. We intend to find out the effect of the layout and number of piezoelectric actuators on the power consumption.

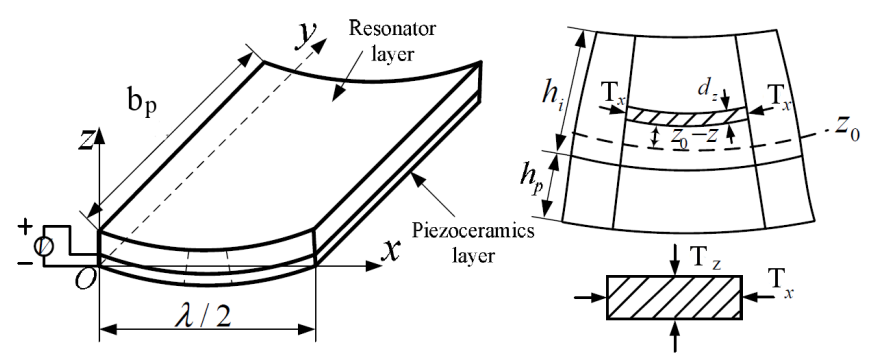

Fig. 4. A half-wavelength section of the bended tactile plate; $h_{i}$ and $h_{p}$ are the thickness of the resonator layer and piezoceramics layer respectively.

\subsection{Plate deflection}

The aim of this paragraph is to calculate the strain of the plate when it vibrates, as a function of the electrical field applied, in order to determine further the power consumption. First, a static analysis will be performed, and second, a dynamic amplification ratio will be introduced. When vibrating at the resonance frequency, the tactile plate creates a uniform standing wave across the plate. For the analytical approach, we consider the bond section of the piezoceramic layer and the resonator with the width of $\lambda / 2$ and the length of $b$. The deflection is analyzed based on Euler-Bernoulli beam theory [13].

The cross section of the bended tactile plate is presented in Fig 4. It is the assembly of the piezoceramics layer and the resonator layer. The two layers are glued together. We assume this assembly is perfect, which leads to consider that the thickness of adhesive is zero and that the strains are continuous on the resonator-piezoceramic interface.

When calculating the bending stress, we assume that cross sections of the plate remain plane during bending, so that they undergo only a rotation with respect to the neutral plane. In Fig.4, the position of the neutral plane is located at the position $z_{0}$. In this paper, the neutral plane is supposed to be be equal to the middle plane. Moreover, the curvature of the deflection curve is assumed to be $\mathrm{d}^{2} w / \mathrm{d} x^{2}$, which equals to the inverse of the radius of curvature, $1 / \rho$. The deflection, $w$, is assumed to be small as compared with the width of the plate, $\lambda / 2$. Then the strain of a fiber parallel to the $x$ axis, $S_{x}$, is proportional to its distance from the middle surface:

$$
S_{x}=\frac{z_{0}-z}{\rho}=\left(z_{0}-z\right) \frac{\mathrm{d}^{2} w}{\mathrm{~d} x^{2}}
$$

According to Hooke's law, the relationship between strain, $S$, and stress, $T$, acting on the shaded element shown in Fig. 4 are:

$$
\begin{gathered}
S_{x}=\frac{T_{x}}{G_{x}}-\nu \frac{T_{y}}{G_{y}} \\
S_{y}=\frac{T_{y}}{G_{y}}-\nu \frac{T_{x}}{G_{x}}=0
\end{gathered}
$$


where $G$ and $\nu$ are Young's module and Poisson's ratio respectively. In our case, the resonator is considered to be isotropic, so $G_{x}=G_{y}=G$. Moreover, the lateral strain in the $y$ direction must be zero in order to maintain continuity in the plate during bending. Substituting 4 into 3 , we obtain:

$$
T_{x}=\frac{G S_{x}}{1-\nu^{2}}=\frac{G}{1-\nu^{2}}\left(z_{0}-z\right) \frac{\mathrm{d}^{2} w}{\mathrm{~d} x^{2}}
$$

Substituting the material properties of the resonator into 5 , we obtain:

$$
T_{x}=G^{\prime} S_{x}
$$

where $G^{\prime}=G /\left(1-\nu^{2}\right)$. As for the piezoelectric layer of the plate, the coupling between the mechanical and the electrical part of the system is described by the constitutive relationships between the stress, strain, and applied electrical fields in terms of the piezoelectric stress relations:

$$
\begin{aligned}
& T=c^{E} S-e^{t} E \\
& D=e S+\varepsilon^{S} E
\end{aligned}
$$

where $E$ and $D$ are, respectively, the electric field intensity vector and the electric displacement vector. The terms $c^{E}, e$, and $\varepsilon^{S}$ are, respectively, the elastic constants short circuit matrix, the voltage coefficient matrix, and the dielectric constants matrix. Superscipt ${ }^{E}$ means at constant dielectric field, and ${ }^{S}$ at constant strain.

Thus, by introducing the matrix coefficients $c_{11}^{E}$ and $e_{31}$ of $c^{E}$ and $e^{t}$, the stress distribution across the plate due to an applied electric field in the z-direction can be summarized as:

$$
T_{x}(z)= \begin{cases}G^{\prime} S_{x}(z) & h_{p} \leq z \leq\left(h_{p}+h_{i}\right) \\ c_{11}^{\prime} S_{x}(z)-e_{31} E_{z} & 0 \leq z \leq h_{p}\end{cases}
$$

where $c_{11}^{\prime}=c_{11} /\left(1-\nu_{p}^{2}\right), E_{z}$ is the electric field in the z-axis. The moment yielded by the piezoceramic causes the resonator to bend. This relationship can be described by:

$$
\int_{h_{p}}^{h_{p}+h_{i}}\left(z_{0}-z\right) T_{x}(z) b \mathrm{~d} z=\int_{0}^{h_{p}}\left(z_{0}-z\right) T_{x}(z) b \mathrm{~d} z
$$

Substituting 2 and 8 into 9 , we can obtain:

$$
\begin{aligned}
\int_{h_{p}}^{h_{p}+h_{i}} & \frac{\left(z_{0}-z\right)^{2}}{\rho} G^{\prime} \mathrm{d} z= \\
& \int_{0}^{h_{p}} \frac{\left(z_{0}-z\right)^{2}}{\rho} c_{11}^{\prime} \mathrm{d} z-\int_{0}^{h_{p}}\left(z_{0}-z\right) e_{31} E_{z} \mathrm{~d} z
\end{aligned}
$$

By integrating 10, we deduce that the deflection curvature can be expressed as follows:

$$
\frac{1}{\rho}=\frac{\mathrm{d}^{2} w}{\mathrm{~d} x^{2}}=\frac{3}{2} \frac{d_{31} E_{z}}{h_{p} a}
$$

where

$$
\frac{1}{a}=\frac{1-2 f_{0}}{1-3 f_{0}+3 f_{0}^{2}-\alpha\left(3 \beta+3 \beta^{2}+\beta^{3}-6 f_{0}-3 \beta f_{0}+3 \beta f_{0}^{2}\right)}
$$

and

$$
\alpha=\frac{G^{\prime}}{c_{11}^{\prime}}, \beta=\frac{h_{i}}{h_{p}}, d_{31}=\frac{e_{31}}{c_{11}^{\prime}}, f_{0}=\frac{z_{0}}{h_{p}}
$$

The above equations calculate the deflection in a static model. At resonance, the strain is enhanced by a dynamic amplification factor, $Q$. It should be noted that this dynamic amplification factor is notthe quality factor. It is used to denote the ratio between the vibration amplitude at resonance and the static amplitude[30]. Moreover, according to our measurement, the strain is also amplified by the number of actuated piezoceramics, $n_{c}$ (see 1). Therefore, the dynamic strain is deduced as:

$$
S_{x}=n_{c} Q \frac{3 d_{31}\left(z_{0}-z\right)}{2 h_{p} a} E_{z}
$$

\subsection{Power calculation}

The power losses $P_{l}$ in the piezoceramics are assumed to include two parts:

$$
P_{l}=P_{m}+P_{d}
$$

where $P_{m}$ and $P_{d}$ represents the mechanical part and the electrical part of the power losses in the piezoelectric layer respectively. These two powers are deduced as how the real power is defined [31]:

$$
\begin{aligned}
& P_{m}=\iiint \frac{1}{2} \operatorname{Re}\left(T \cdot \dot{S}^{*}\right) \mathrm{d} V \\
& P_{d}=\iiint \frac{1}{2} \operatorname{Re}\left(E \cdot \dot{D}^{*}\right) \mathrm{d} V
\end{aligned}
$$

where $\dot{S}^{*}$ and $\dot{D}^{*}$ are the conjugates of the derivative terms $\dot{S}$ and $\dot{D}$ respectively. As shown in Fig. 4, the piezoceramic layer is powered in the z-direction and it yields a movement along the $\mathrm{x}$-direction. According to 8 and 14, the stress in the piezoelectric layer is:

$$
\begin{aligned}
T_{x} & =c_{11}^{\prime} S_{x}-e_{31} E_{z} \\
& =n_{c} Q c_{11}^{\prime} \frac{3 d_{31}\left(z_{0}-z\right)}{2 h_{p} a} E_{z}-d_{31} c_{11}^{\prime} E_{z}
\end{aligned}
$$

The electric displacement is written as:

$$
\begin{aligned}
D_{z} & =e_{31} S_{x}+\varepsilon_{33} E_{z} \\
& =n_{c} Q c_{11}^{\prime} \frac{3 d_{31}^{2}\left(z_{0}-z\right)}{2 h_{p} a} E_{z}+\varepsilon_{33} E_{z}
\end{aligned}
$$


where $\varepsilon_{33}$ is the dielectric constant in z-direction. Taking the Laplace transform of $\dot{S}_{x}$ and $\dot{D}$, we can obtain their forms in the frequency domain:

$$
\begin{gathered}
\dot{S}_{x}=n_{c} Q \frac{3}{2} \frac{d_{31}\left(z_{0}-z\right)}{2 h_{p} a} j \omega E_{z} \\
\dot{D}_{z}=\left(n_{c} Q c_{11}^{\prime} \frac{3}{2} \frac{d_{31}^{2}\left(z_{0}-z\right)}{2 h_{p} a}+\varepsilon_{33}\right) j \omega E_{z}
\end{gathered}
$$

Since we found that piezoceramics only cost power when they were powered with voltage, we assumed that the power consumption was caused by hysteresis losses in piezoelectric coupling [29]. Therefore, a complex parameter, $\bar{d}_{31}$, is introduced to calculate the power losses:

$$
\bar{d}_{31}=d_{31}\left(1-j \tan \theta^{\prime}\right)
$$

where $\theta^{\prime}$ is the phase delay of the strain when an electric field is applied, or the phase delay of the electric displacement when a stress is applied.

Substitute the complex piezoelectric constant $d_{31}$ in 18, 20 and 21, we can calculate the mechanical part of power losses as:

$$
\begin{gathered}
P_{m}=\iiint \frac{1}{2} \mathrm{R}_{\mathrm{e}}\left(d _ { 3 1 } ( 1 - j \operatorname { t a n } \theta ^ { \prime } ) \left[n_{c} Q c_{11}^{\prime} \frac{3}{2} \frac{\left(z_{0}-z\right)}{2 h_{p} a} E_{z}\right.\right. \\
\left.\left.-c_{11}^{\prime} E_{z}\right] \cdot n_{c} Q \frac{3}{2} \frac{d_{31}\left(1+j \tan \theta^{\prime}\right)\left(z_{0}-z\right)}{2 h_{p} a}(-j) \omega E_{z}\right) \mathrm{d} V
\end{gathered}
$$

Since $d_{31}\left(1-j \tan \theta^{\prime}\right) \cdot d_{31}\left(1+j \tan \theta^{\prime}\right) \cdot(-j)=-j\left\|d_{31}^{\prime}\right\|^{2}$ is not a real number, there is no real part in 23. As a result, the mechanical part of power losses in the piezoelectric layer equals to zero.

Similarly, we can also calculate the electrical part of power losses, in a domain restricted to the piezoelectric actuators:

$$
\begin{gathered}
P_{d}=n_{c} \int_{x=0}^{x=\lambda / 2} \int_{y=0}^{y=b_{p}} \int_{z=0}^{z=h_{p}} \frac{1}{2} \mathrm{R}_{\mathrm{e}}\left(E _ { 3 } \left(n_{c} Q c_{11}^{\prime}\right.\right. \\
\left.\left.\frac{3}{2} \frac{d_{31}^{2}\left(1+2 j \tan \theta^{\prime}+\tan \theta^{\prime 2}\right)\left(z_{0}-z\right)}{2 h_{p} a}+\varepsilon_{33}\right)(-j) \omega E_{3}\right) \mathrm{d} V
\end{gathered}
$$

We calculate the real power, leading to:

$$
P_{d}=V^{2} n_{c}^{2} Q \tan \theta^{\prime} \frac{3 b_{p} \lambda}{4} \pi f d_{31}^{2} c_{11}^{\prime}\left(\frac{2 z_{0}-h_{p}}{h_{p}^{2} a}\right)
$$

where $f$ is the vibration frequency, $b_{p}$ is the length of a piece of piezoceramics.

According to this model, the power losses are in proportion to the square of the number of piezoceramics and the applied voltage. This result is consistent with our measurements (see Table 4). In our application, the plate should vibrate at ultrasonic frequency in order to generate the squeeze air film effect. Moreover, as compared with the applied voltage, we care more about the vibration amplitude which should be above $1 \mu m$ [13]. Therefore, we need to deduce the relationship between the power and the vibration amplitude.

The dynamic displacement profile of the simply supported beam can be obtained by integrating 11 twice and multiply the dynamic amplification factor and the number of powered piezoceramics:

$$
w(x)=Q n_{c} \frac{3}{4} \frac{d_{31} V}{h_{p}^{2} a}\left(x^{2}-\frac{\lambda}{2} x\right)
$$

The vibration amplitude at $\lambda / 4$ is calculated as:

$$
A=Q n_{c} \frac{3}{64} \frac{d_{31} V}{h_{p}^{2} a} \lambda^{2}
$$

Then, we can deduce the power losses using only the vibration amplitude A by substituting 27 to 25 :

$$
P_{l}=\frac{A^{2}}{Q} \tan \theta^{\prime} f b_{p} c_{11}^{\prime}\left(2 z_{0}-h_{p}\right) \frac{1024 h_{p}^{2} a}{3 \lambda^{3}}
$$

The above equation indicates that the power is in proportion with the square of vibration amplitude. This result has also been proved by our measurement results, as shown in Table 4. In our application of providing tactile feedback, we need the device to work at constant vibration amplitude rather than at constant voltage [22]. According to 28 , when vibration amplitude is constant, the power consumption is independent of the number of piezoceramics. Namely, we can use very few piezoelectric actuators to excite the resonator while costing the same amount of power. The reduction of piezoceramics will reduce the cost and weight of the tactile plate. This result is significant for the design of a large but compact tactile device. However, according to (28), the power consumption depends on the dynamic amplification factor, $Q$. Therefore, in the following section, we discuss the parameters that influence this factor.

\section{Effect of the actuator's layout}

$\mathrm{d} V$ The values of two parameters in (28 are unknown. One ( 2 is the piezoelectric loss tangent, $\tan \theta^{\prime}$. The other is the dynamic amplification factor, $Q$. To obtain these data, we made six small tactile plate samples with different actuator layout and resonator thickness. We intended to use the data obtained from the small plates to estimate the power consumption of large tactile plates. Fig. 5 shows the aluminum resonators with different layouts of the piezoceramics. The dimension of these aluminum resonators is $76 \mathrm{~mm} \times 41 \mathrm{~mm}$, varying in thickness $(2 \mathrm{~mm}$ and $1.2 \mathrm{~mm})$. The size of piezoceramics is $5 \mathrm{~mm} \times 9 \mathrm{~mm} \times 0.5 \mathrm{~mm}$.

The dynamic amplification factor $Q$ is calculated from 27 with the measurement results of vibration amplitude and applied voltage. The piezoelectric loss tangent is calculated by dividing $Q$ from the product of $Q \tan \theta^{\prime}$. This product is obtained from 25 with the measurement results of power consumption and applied voltage. We found that the layout of the piezoceramics had a significant effect on 


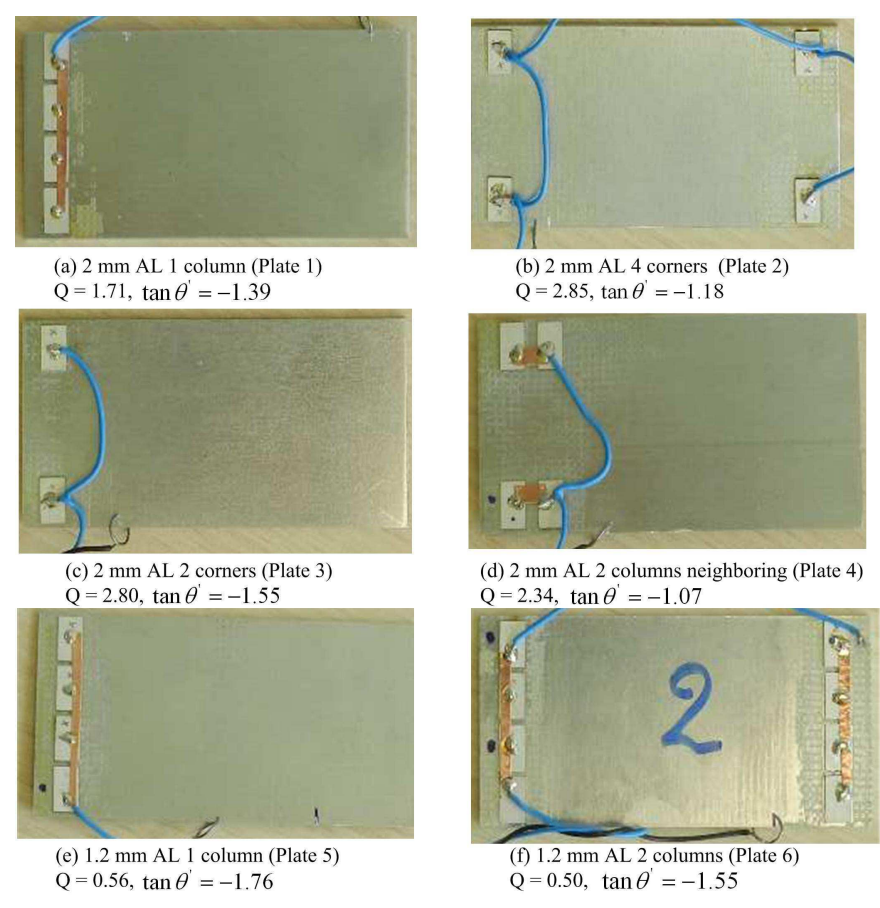

Fig. 5. Aluminum resonators with different piezoceramics layouts. The dynamic amplification factor $(Q)$ and the piezoelectric loss tangent $\left(\tan \theta^{\prime}\right)$ are labeled in each condition.

the dynamic amplification factor, $Q$. Comparing Plate 1 with Plate 2 and Plate 4 (that have the same number of piezoceramics), we found that the farther the piezoceramics are from each other, the higher the dynamic amplification factor. Therefore, we conclude that piezoceramics should not be placed close to each other in order to increase the dynamic amplification factor.

Nevertheless, we did not find a clear relationship between the layout of the piezoceramics and the $\tan \theta^{\prime}$ value. However, we found that generally it varies with the thickness of the resonator. The mean $\tan \theta^{\prime}$ with $2 \mathrm{~mm}$ resonator is -1.30 while that with the $1.2 \mathrm{~mm}$ is -1.64 . This result was not expected since $\tan \theta^{\prime}$ should be a characteristic of the piezoceramic itself. At least if there is no measurement error, one assumption to explain this result may be that the loss tangent varies since the stress on piezoceramics changed [29] due to the variation of neutral surface position as a result of different resonator thickness.

We also noticed that duplicate the same layout of actuators of each column to a column that is far away does not change the dynamic amplification factor (see Plate 3 and Plate 2, Plate 5 and Plate 6) even if the number of piezoceramics is doubled. This result indicates that we can place piezoceramic at the two opposite edges of the resonator if we need to put more actuators on the plate. This design will not deteriorate the dynamic amplification factor.

\section{Design of a large tactile device}

We have developed an analytical model to estimate the power consumption of the tactile plate. According to this model, when vibration amplitude is constant, the power consumption of the tactile plate is irrelevant to the number of piezoceramic actuators. However, we noticed that the layout of the piezoceramic actuators affects the dynamic amplification factor significantly. Optimal layout is realized by placing the piezoceramics far away from each other, for instance, placing them at the four corners of the resonator. These findings offer guidelines for the design of a large but compact tactile plate. In the following section, we present the design of the large tactile plate and its power measurement to validate the analytical power modeling.

\subsection{Design guidelines}

We intend to design a large but portable tactile interface which is able to provide programmable friction coefficient. Therefore, the design should satisfy several requirements considering different aspects. First of all, the tactile plate is based on the squeeze air film effect. The squeeze number should be above 10 so as to realize a perceivable tactile feedback [13]. As a result, we need to excite the plate at an ultrasonic frequency $(>25 k \mathrm{~Hz})$ with an amplitude above $1 \mu m$ [13]. Secondly, ultrasonic vibration modes exhibit nodal lines. Along nodal lines, squeeze film effect is diminished. To minimize this effect, we use a standing wave mode shape with the half wavelength similar to the width of fingertip [17]. Thirdly, we intend to design a large tactile plate whose workspace is similar to that of mainstream consumer electronics such as the Apple IPad. Finally, we use the minimum number of piezoelectric actuators by placing them following the optimal layout to reduce cost and power consumption. These requirements are summarized as follows,

a) Squeeze air film effect: resonance frequency $>25 \mathrm{kHz}$, vibration amplitude $>1 \mu \mathrm{m}$,

b) Mode shape: uniform standing wave, half wave length $\lambda / 2 \leq 15 \mathrm{~mm}$,

c) Actuator layout: gluing piezoceramics at the four corners of the resonator.

\subsection{Implementation}

The resonator is made of aluminum for its light weight and easy manufacture. The size is $198 \mathrm{~mm} \times 138 \mathrm{~mm}$. The thickness of the resonator should be as thin as possible to minimize power consumption [22]. However, the tactile plate must also have sufficient rigidity to prevent from any deformation during user's exploration. Therefore, we use the $2 \mathrm{~mm}$ thick resonator as a trade-off between mechanical resistance and power consumption. Moreover, the $2 \mathrm{~mm}$ thick resonator can produce a high frequency (above $50 \mathrm{kHz}$ ) standing wave mode so that the user cannot hear 


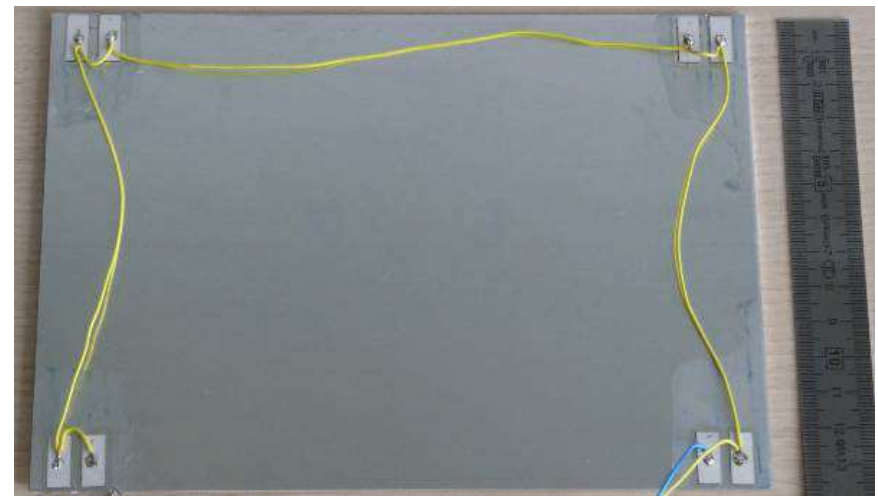

Fig. 6. The layout of the eight piezcoceramics on the large tactile plate.

the noise when it works. It may be noted that we did not use glass resonator in this prototype. The first reason is that our primary motivation in this research is to validate our power modeling rather than developing a tactile touch screen. Secondly, the aluminum resonator is easy to manufacture. However, it is possible to replace this resonator with transparent ones, such as glass [17] and off-the-shelf capacitance touch screens [32].

Eight PZT piezoceramics (NCE 41, Noliac Inc.) are glued at the backside of the resonator, as shown in Fig. 6 . The half wavelength is designed to be $9 \mathrm{~mm}$. The piezoceramics are chosen with dimension of $6 \mathrm{~mm} \times 14 \mathrm{~mm} \times$ $0.5 \mathrm{~mm}$. The piezoceramics are placed equally at the four corners of the resonator to minimize the power consumption. In fact, we can use only four pieces of them. However, the fewer number of piezoelectric plates we use, the higher voltage is needed to excite the plate (see 27). Moreover, we also need one piece of the piezoceramics as a vibration sensor to set up a close-loop control of the vibration amplitude. Therefore, we glue extra four pieces of piezoceramics symmetrically on the resonator. To minimize their effects on the dynamic amplification factor, they are glued as the mode in Plate 4 (Fig. 5). The material properties are listed in Table 2.

\subsection{Experimental measurements}

We conducted several measurements to validate the design and the power modeling. The measurement setup is similar to the one we presented in Section 2.1 of this paper. Before the measurements, we adjusted the waveform generator to activate the plate at the desired resonance frequency. We then applied several voltages to the plate and measured its vibration amplitude with a Laser Doppler Vibrometer. The power consumption and the vibration velocity of the plate were read from an oscilloscope.

\subsubsection{Mode shape and vibration amplitude}

The expected $(21,0)$ mode shape was found at the resonance frequency of $52.4 \mathrm{kHz}$. To further confirm the mode

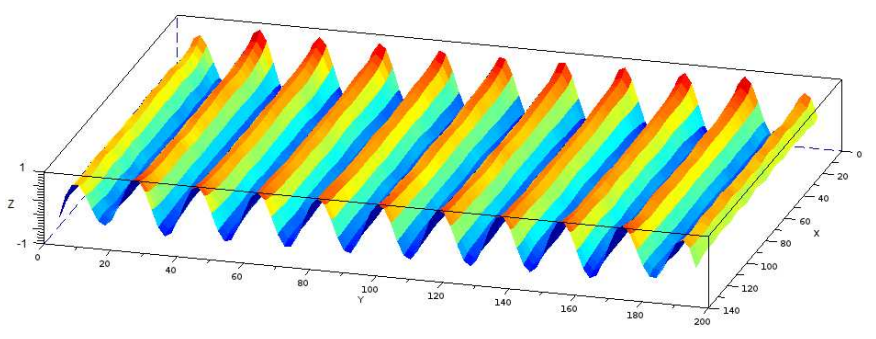

Fig. 7. Cartography of the large tactile plate.

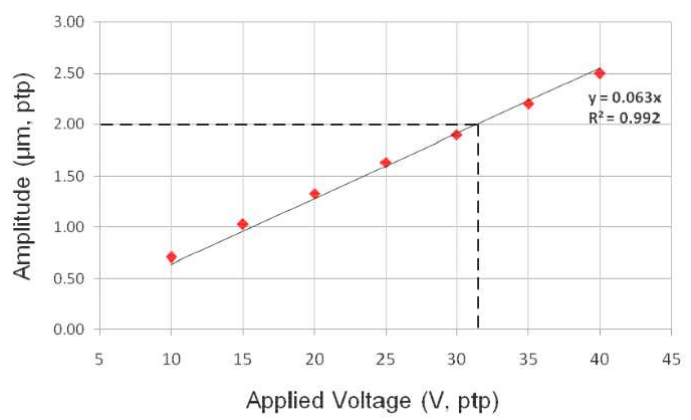

Fig. 8. Vibration amplitude as a function of applied voltage..

shape, we made cartography of the plate. The tactile plate was fixed on a servo-controlled x-y platform. The platform moved with a step of $2 \mathrm{~mm}$ along either the $\mathrm{x}$ or $\mathrm{y}$ direction. We measured and record the vibration amplitude of each sample point of the plate and made the cartography, as shown in Fig. 7.

The cartography indicates that the mode shape is uniform across the plate. This shape is good to provide an homogeneous tactile feedback to the users. Then, we measured the relationship between vibration amplitude and the applied voltage. The measurement was carried out at the center of the plate since this area was explored most frequently. The measurement result is presented in Fig. 8. The vibration amplitude has a linear relationship with the applied voltage. This result agrees with our model in 27 .

\subsubsection{Power consumption measurement}

The power consumption of the large tactile device is presented in Fig. 9. The power has a linear relationship with the square of voltage as analyzed in our modeling (Eq. 25). According to Fig. 8 and Fig. 9, we can infer that this tactile plate needs only $1.3 \mathrm{~W} @ 32 \mathrm{~V}$ ptp to excite a perceivable friction reduction with the vibration amplitude above $1 \mu m$.

In addition to the measurement, we also find a connection between the measurement results from the large tactile plate with that of the small ones. Firstly, the loss tangent has a similar value when the thickness of the resonator is the same (1.30 (small) Vs. -1.24 (large)). Secondly, the dynamic amplification factor is predictable when piezoceramics have the same layout. For example, the layout of the large tactile plate is similar to the one of Plate 4 (Fig. 5), by duplicating four groups of piezoceramics at 


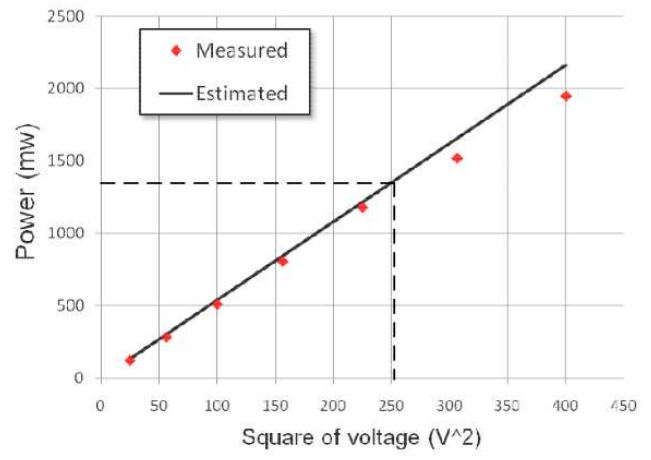

Fig. 9. Measured and estimated power as a function of the square of voltage.

Table 5. The measured and estimated dynamic amplification factor and piezoelectric loss tangent.

\begin{tabular}{lcc}
\hline & Estimated & Measured \\
$Q_{\text {Large }}$ & 1.51 & 1.48 \\
$\tan \theta^{\prime}$ & -1.30 & -1.24 \\
\hline
\end{tabular}

the four corners. So we should have found an amplification factor around 2.34. However, the length of the piezoceramics on the large tactile plate is $14 \mathrm{~mm}$ while that of the small ones is $9 \mathrm{~mm}$. This factor may also influence the $Q$ value. If we come back to the results shown in Fig. 5 for the plates 1 and 3, we can remark that, considering the two neighboring piezoceramics in Plate 1 as one which has twice the length, the $Q$ factor is lower when the length of the piezoceramics is higher. For our plates, we assume this effect is linear and we propose the following relation:

$$
Q_{\text {Large }}=Q_{\text {Small }} \frac{b_{\text {Large }}}{b_{\text {Small }}}
$$

Where $Q_{\text {Large }}$ is the dynamic amplification factor obtained from the small plates, $b_{\text {small }}=9 \mathrm{~mm}$ is the length of the piezoelectric plate used in the small plates, $b_{\text {large }}=$ $14 \mathrm{~mm}$ is the length of the piezoelectric plate used in the large plate. In this case, we find that the dynamic amplification factor can be calculated from 29 is consistent with the measurement result of Table 5 .

With these data, we can estimate the power consumption of the large tactile plate according to 25 . The estimated and measured powers as a function of the square of voltage are presented in Fig.9. The estimated power consumption is very close to the measured data with an average error less than $10 \%$. Of course, although this is a satisfactory result, we still need more measurements to define the limitations of the extrapolation from small plates to large ones.

\section{Conclusion}

We present the power analysis of an ultrasonic tactile device for purpose of enlarging its workspace. In the first step, we conducted a series of measurements to investigate the source of the power consumption. We find the power is mainly from the internal behavior of the powered piezoelectric actuators. We then developed an analytical model based on the piezoelectric hysteresis to estimate the power. The model shows that, when the vibration amplitude is constant, the power consumption is not related to the number of piezoelectric actuators but related to their layout. The optimal layout is to place the piezoelectric actuators far away from each other, e.g., at the four corners of the resonator. According to this result, we designed a large area tactile plate $(198 \mathrm{~mm} \times 138 \mathrm{~mm})$ with only eight piezoelectric actuators. The device needed only $1.3 W @ 32 \mathrm{~V}$ ptp to excite a perceivable friction reduction with the vibration amplitude at $1 \mu \mathrm{m}$. Moreover, we also find that the power consumption was predictable with the measurement results from the small plates with an average error of less than $10 \%$.

This research provides guidelines for our future work to develop a tactile touch screen by replacing the aluminum resonator by a transparent one. Psychophysics experiments to evaluate users' feedback on the large tactile plate will be undertaken to further improve the device.

\section{References}

1. M. Levin and A. Woo. Tactile-feedback solutions for an enhanced user experience. Information Display, 25:18-21, 2009.

2. B. Banter. Touch screens and touch surfaces are enriched by haptic force-feedback. Information Display, 26:26-30, 2010 .

3. Stephen Brewster, Faraz Chohan, and Lorna Brown. Tactile feedback for mobile interactions. In Proceedings of the SIGCHI Conference on Human Factors in Computing Systems, CHI '07, pages 159-162, 2007.

4. Masaaki Fukumoto and Toshiaki Sugimura. Active click: Tactile feedback for touch panels. In CHI '01 Extended Abstracts on Human Factors in Computing Systems, CHI EA '01, pages 121-122, New York, NY, USA, 2001.

5. Seung-Chan Kim, Tae-Hon Yang, Byung-Kil Han, and Dong-Soo Kwon. Interaction with a display panel - an evaluation of surface-transmitted haptic feedback. In Control, Automation and Systems, 2008. ICCAS 2008. International Conference on, pages 278-283, Oct 2008.

6. Ivan Poupyrev, Shigeaki Maruyama, and Jun Rekimoto. Ambient touch: Designing tactile interfaces for handheld devices. In Proceedings of the 15th Annual ACM Symposium on User Interface Software and Technology, UIST '02, pages 51-60, 2002.

7. P. Laitinen and J. Maenpaa. Enabling mobile haptic design: piezoelectric actuator technology properties in hand held devices. In Haptic Audio Visual Environments and their Applications, 2006. HAVE 2006. IEEE International Workshop on, pages 40-43.

8. Kaoru Tashiro, Yuta Shiokawa, Tomotake Aono, and Takashi Maeno. A virtual button with tactile feedback using ultrasonic vibration. In Virtual and Mixed Reality, volume 5622 of Lecture Notes in Computer Science, pages 385-393. Springer Berlin Heidelberg, 2009.

9. Yvonne Jansen, Thorsten Karrer, and Jan Borchers. Mudpad: Tactile feedback and haptic texture overlay for touch 
surfaces. In ACM International Conference on Interactive Tabletops and Surfaces, pages 11-14, 2010.

10. Chris Harrison and Scott E. Hudson. Providing dynamically changeable physical buttons on a visual display. In Proceedings of the SIGCHI Conference on Human Factors in Computing Systems, CHI '09, pages 299-308, 2009.

11. Olivier Bau, Ivan Poupyrev, Ali Israr, and Chris Harrison. Teslatouch: Electrovibration for touch surfaces. In Proceedings of the 23Nd Annual ACM Symposium on User Interface Software and Technology, UIST '10, pages 283292, 2010.

12. H. Kotani, M. Takasaki, and T. Mizuno. Surface acoustic wave tactile display using a large size glass transducer. In Mechatronics and Automation, 200\%. ICMA 200\%. International Conference on, pages 198-203, Aug 2007.

13. M. Biet, F. Giraud, and B. Lemaire-Semail. Squeeze film effect for the design of an ultrasonic tactile plate. Ultrasonics, Ferroelectrics, and Frequency Control, IEEE Transactions on, 54(12):2678-2688, December 2007.

14. T. Watanabe and S. Fukui. A method for controlling tactile sensation of surface roughness using ultrasonic vibration. In Robotics and Automation, 1995. Proceedings., 1995 IEEE International Conference on, volume 1, pages 1134-1139 vol.1, May 1995.

15. L. Winfield, J. Glassmire, J.E. Colgate, and M. Peshkin. T-pad: Tactile pattern display through variable friction reduction. In EuroHaptics Conference, 2007 and Symposium on Haptic Interfaces for Virtual Environment and Teleoperator Systems. World Haptics 200\%. Second Joint, pages 421-426, March 2007.

16. N.D. Marchuk, J.E. Colgate, and M.A. Peshkin. Friction measurements on a large area tpad. In Haptics Symposium, 2010 IEEE, pages 317-320, March 2010.

17. F. Giraud, M. Amberg, B. Lemaire-Semail, and G. Casiez. Design of a transparent tactile stimulator. In Haptics Symposium (HAPTICS), 2012 IEEE, pages 485-489, March 2012.

18. M. Biet, G. Casiez, F. Giraud, and B. Lemaire-Semail. Discrimination of virtual square gratings by dynamic touch on friction based tactile displays. In Haptic interfaces for virtual environment and teleoperator systems, 2008. haptics 2008. symposium on, pages 41-48, March 2008.

19. G. Casiez, N. Roussel, R. Vanbelleghem, and title = Surfpad: Riding Towards Targets on a Squeeze Film Effect booktitle = Proceedings of the SIGCHI Conference on Human Factors in Computing Systems series $=$ CHI '11 year $=2011$ isbn $=$ 978-1-4503-0228-9 location = Vancouver, BC, Canada pages $=2491-2500$ Giraud, F.

20. Vincent Levesque, Louise Oram, Karon MacLean, Andy Cockburn, Nicholas D. Marchuk, Dan Johnson, J. Edward Colgate, and Michael A. Peshkin. Enhancing physicality in touch interaction with programmable friction. In Proceedings of the SIGCHI Conference on Human Factors in Computing Systems, CHI '11, pages 2481-2490, 2011.

21. Joe Mullenbach, Craig Shultz, Anne Marie Piper, Michael Peshkin, and J. Edward Colgate. Surface haptic interactions with a tpad tablet. In Proceedings of the Adjunct Publication of the 26th Annual ACM Symposium on User Interface Software and Technology, UIST '13 Adjunct, pages 7-8, 2013.

22. F. Giraud, M. Amberg, R. Vanbelleghem, and B. LemaireSemail. Power consumption reduction of a controlled friction tactile plate. In Haptics: Generating and Perceiving
Tangible Sensations, volume 6192 of Lecture Notes in Computer Science, pages 44-49. Springer Berlin Heidelberg, 2010.

23. Biet M., Giraud F., and Semail B. Vibrating tactile interface. European Patent, EP1956466, Aug 2008.

24. M. Wiertlewski and J.E. Colgate. Power optimization of ultrasonic friction-modulation tactile interfaces. Haptics, IEEE Transactions on, 8(1):43-53, Jan 2015.

25. Sergeant P., Giraud F., and Lemaire-Semail B. Geometrical optimization of an ultrasonic tactile plate. Sensors and Actuators A: Physical, 161(12):91 - 100, 2010.

26. K. Uchino, J.H. Zheng, Y.H. Chen, X.H. Du, J. Ryu, Y. Gao, S. Ural, S. Priya, and S. Hirose. Loss mechanisms and high power piezoelectrics. In Frontiers of Ferroelectricity, pages 217-228. 2007.

27. Noliac. Available: http://www.noliac.com/Specification141.asp.

28. T. L. Jordan and Z. Ounaies. Piezoelectric ceramics characterization. Technical report, NASA, 2001.

29. K. Uchino and S. Hirose. Loss mechanisms in piezoelectrics: how to measure different losses separately. Ultrasonics, Ferroelectrics, and Frequency Control, IEEE Transactions on, 48(1):307-321, Jan 2001.

30. Ahid D. Nashif and I. G. Jones John P. Henderson. Vibration Damping. Wiley-Interscience, 1985.

31. A.V. Mezheritsky. Elastic, dielectric, and piezoelectric losses in piezoceramics: how it works all together. Ultrasonics, Ferroelectrics, and Frequency Control, IEEE Transactions on, 51(6):695-707, June 2004.

32. M. Amberg, F. Giraud, B. Semail, P. Olivo, G. Casiez, and N. Roussel. Stimtac: A tactile input device with programmable friction. In Proceedings of the 24th Annual ACM Symposium Adjunct on User Interface Software and Technology, UIST '11 Adjunct, 2011. 